\title{
EL RELATIVISMO DEL CONOCIMIENTO ${ }^{1}$
}

Julián Urrea B. Profesor Asociado Depto. Física, Universidad Pedagógica Nacional e-mali: jurrea@zeus.uniandes.edu.co

\begin{abstract}
Facing countercultural thought of sujective reasoning and submission of scientific truth to social, political and cultural concerns, a cate ful judgement of mainstream theories of science, particularly of physics, points toward an epistemology in which events in the physical world occur independently of observation, and the limits and validity of science are independent of social and cultural conditions.
\end{abstract}

\section{RESUMEN}

Frente a la contracultura del pensamiento subjetivo y de la subordinación de la verdad científica a causas sociales, políticas y culturales, el análisis crítico de las teorías dominantes de la ciencia en general y de la física en particular, sugiere una visión epistemológica en que los hechos del mundo físico tienen entidad propia, distinta e independiente del sujeto y el alcance y la validez de los enunciados de las ciencias son independientes del medio, de la cultura y de la época.

\section{INTRODUCCIÓN}

La evidencia de las leyes de la Física proviene de la coincidencia entre las predicciones teóricas y nuestras percepciones del mundo y de la continuidad entre éste último y el mundo de la percepción.

Sin embargo el proceso que conduce a la percepción a partir de la predicción es impreciso, quizás ajeno a la causalidad, aunque, según el sentido común, de algún modo es la percepción la que nos da a conocer directamente el mundo exterior.

En consecuencia también hay alguna imprecisión acerca de la "verdad' de la física, aunque es posible decir que la física es "verdadera' si percibimos un objeto de conformidad con alguna de sus predicciones. La evidencia de la verdad física es, pues, empírica.

Sin embargo los epistemólogos de la física sostienen, unos, que el mundo físico sólo es el mundo de la percepción, otros, que el mundo físico existe tal como nos lo revela la percepción, e independientemente de ella. En términos más precisos, el problema consiste en dirimir si existen o no estructuras simples o complejas que satisfacen las hipótesis de las que se deriva la física como sistema deductivo.

Una primera aproximación a la solución del problema consiste en aceptar que los antecedentes relacionados causal-mente con nuestras percepciones pueden no ser

\footnotetext{
${ }^{1}$ Proyecto de investigación Las matemáticas en su relación con la física: una propuesta didáctica dentro del nuevo piando estudios de licenciatura en física. Financiado por el CIUP.
} 
percepciones. En la práctica, este ha sido el punto de vista de la mayoría de los físicos (Russel, 1976) y de notables maestros de la física.

Como sistema deductivo, las leyes de la Física se construyen por medio de razonamientos matemáticos a partir de axiomas o postulados. En este sentido las leyes de la Física son, además, analíticamente verdaderas y pueden considerarse como el resultado de un compromiso entre la verdad empírica y la verdad lógica. A este doble carácter hace referencia el concepto de verdad científica (LeShan y Margenau, 1996).

Frente al problema del conocimiento, en general y del conocimiento científico en particular, se han planteado distintas posiciones epistemológicas. El espectro se extiende desde el dogmatismo hasta el escepticismo; en otras palabras, desde el desconocimiento del sujeto hasta el desconocimiento del objeto (Hessen, 1997). Algunas van menos lejos, como el subjetivismo, para el que la validez de la verdad está limitada por el "sujeto que conoce y juzga". Su pariente más cercano es el relativismo, para el cual la influencia del medio, de la cultura y de la época son factores externos que delimitan la validez de las verdades de las ciencias naturales. "Sólo hay verdades con relación a una humanidad determinada", es, quizás, la sentencia más conocida en defensa de esta corriente de pensamiento, escrita por Spengler en su obra Decadencia de Occidente.

En una fase inicial de análisis acerca del origen y de las implicaciones que, desde el punto de vista de la enseñanza de las ciencias empíricas, pudiera tener la propuesta relativista, considero particularmente importante una obra, de aparición reciente: Imposturas Intelectuales de A. Sokal y J. Bricmont, ampliamente comentada en círculos científicos y no científicos.

Trataré de presentar aspectos de la crítica que los autores mencionados hacen, en particular, del pensamiento de Thomas kuhn y Paul Feyerabend, quienes, dentro de esa tendencia filosófica, han formulado audaces críticas a la ciencia moderna, en sus obras más conocidas: Estructura de las revoluciones científicas y Contra el método, respectivamente.

\section{EL POSMODERNISMO}

Estudiantes y profesores de las universidades y en general, los intelectuales de hoy, han sido testigos del derrumbamiento de sistemas políticos que parecían invulnerables. No podemos desconocer que bajo su ejercicio de poder y dominación se ocultaron ideales de justicia e igualdad que de un momento a otro perdieron credibilidad, o por lo menos eficacia, frente a los nuevos esquemas de competencia y de libre mercado.

La consecuente desorientación y el desánimo político han generado una actitud confusa y un nuevo esquema de pensamiento, dentro del cual no se trata de enfrentar la realidad sino de perseguir quimeras intelectuales y de evitar la difícil tarea de resolver los grandes problemas que plantean la configuración geopolítica actual y las nuevas estructuras de poder. En opinión de algunos autores, no pocos elementos de este esquema constituyen un puente tendido entre nuevos movimientos sociales y la corriente de pensamiento conocida como posmodernismo.

El posmodernismo es una corriente intelectual que pretende suplantar al pensamiento racionalista de la filosofía moderna. Algunos rasgos, como el escepticismo alrededor de la ciencia actual, la preponderancia de las ideas subjetivas sin importar la verdad o la 
falsedad de sus proposiciones y la supremacía del discurso en relación con los hechos referidos en el mismo, caracterizan la influencia del posmodernismo en las humanidades y en las ciencias sociales.

La referencia a la ciencia como a "una superstición particular" (Feyerabend, 1975), la tendencia a confundir, por ejemplo, la astronomía con la astrología, por parte de algunos y la pretensión, por parte de otros, de que la neurofisiología y la sociobiología desplacen la investigación de las ciencias sociales, ilustran intereses de la investigación en áreas de las ciencias sociales y de las ciencias empíricas.

La realidad, sin embargo, no es tan dramática. Por ejemplo, la química no ha cedido su espacio, como ciencia autónoma, a la física, aunque los fundamentos de la primera se explican íntegramente con la mecánica cuántica. Menos probable, aún, es la desaparición de las ciencias humanas ante el avance de la biología en la explicación del comportamiento humano.

Un aspecto en el que las posturas de intelectuales y científicos son irreducibles es la apropiación por parte de aquellos, de ideas de la filosofía de la ciencia para aplicarlas a causas políticas y sociales. Como consecuencia de esta apropiación indebida, no son escasos los autores que dan prioridad a intereses y puntos de vista subjetivos sobre el contenido objetivo de los hechos y de los datos de la experiencia.

Veamos los términos en que Gilles Deleuze explica cómo pueden conciliar-se lo que él llama los defectos de los sentidos y la existencia de un objeto:

La más pequeña idea, la impresión más pequeña, no es un punto matemático ni un punto físico, sino un punto sensible. El punto físico ya es extenso, y además divisible; el punto matemático es una nada... El punto sensible o átomo es visible y tangible, coloreado y sólido. No tiene extensión por sí mismo y sin embargo existe (Deleuze, 1968).

Este concepto de "objetividad" contrasta con la descripción de "objeto" de L. Von Bertalanffy que leeremos enseguida:

Consideraremos objetos a las entidades a las que se llega en la percepción y son discretas en el espacio y en el tiempo. No dudamos que un guijarro, una mesa,... o una estrella son reales y existen independientemente de la observación. La percepción, sin embargo no es una guía digna de confianza. Siguiéndola "vemos" al sol girar alrededor de la tierra y ciertamente no vemos que un trozo sólido de materia,... es "realmente", en su mayor parte, espacio vacío más unos menudos centros de energía dispersos a distancias astronómicas los unos de los otros (Bertalanffy, 1987).

Podemos aun hallar otra forma de ver el mundo real en R. Carnap cuando dice:

Las leyes de la lógica y de la matemática pura, por su naturaleza misma, no pueden ser utilizadas como base de la explicación científica porque no nos dicen nada que permita diferenciar el mundo real de cualquier otro mundo posible. Cuando preguntamos por la explicación de un hecho, de una observación particular en el mundo real, debemos utilizar leyes empíricas. Estas no poseen la certeza de las leyes lógicas y matemáticas pero nos dicen algo acerca de la estructura del mundo. (Carnap, 1986). 
Es evidente que las dos últimas lecturas sin coincidir entre sí, contrastan con la primera, que puede servir de punto de partida a diversas formas de "relativismo" propuestas en autores como T. Kuhn y $\mathrm{P}$. Feyerabend principalmente y sustentadas, parcialmente al menos, en dos ideas centrales de su pensamiento, conocidas como inconmensurabilidad y subdeterminación de las teorías científicas, según se verá a continuación.

En algunas ocasiones, enseña Kuhn, "la ciencia normal" se ve abocada a un cambio de "paradigma" en un proceso revolucionario provocado por una crisis. En ausencia de crisis, la ciencia normal especifica la clase de problemas, objeto de investigación, los criterios de evaluación de los resultados y el método experimental de verificación que tiene validez.

En el esquema de kuhn, circunscrito a las ideas enunciadas en el párrafo anterior, no hay novedad ni propósito anticientífico alguno. Sin embargo, refiriéndose a la incomensurabilidad de los paradigmas, Kuhn afirma que los científicos tienen la posibilidad de elegir, con base en el experimento, entre teorías que explican los mismos fenómenos desde distintos paradigmas, mecánica clásica y mecánica cuántica, por ejemplo.

Pero si la idea de mundo que el científico acepta es un resultado condicionado radicalmente por su teoría al interior de un paradigma, no es posible comparar racionalmente dos teorías que, desde paradigmas distintos, concurren en el mismo objeto $y$, en consecuencia, la aceptación de una teoría es el resultado de "forzar la naturaleza para que se adapte a ella" (Kuhn, 1990).

Kuhn parece apoyar esta conclusión, entre otras cosas, en el hecho de que, a partir de Dalton, los químicos aceptaron razones de números enteros, en substitución de números decimales, para determinar las proporciones en los compuestos químicos, con base en que, "muchos de los datos de la época se adecuaban a la teoría atómica", ignorando en todo caso, según él "que algunos experimentos arrojaban resultados conflictivos" (Sokal y Bricmont 1999).

La subdeterminación, también conocida como "tesis de Duhen-Quine", es referida por quienes la sustentan al hecho de que las teorías están "subdeterminadas por los hechos" (Sokal y Bricmont, 1999) que, en conjunto proporcionan solamente un número finito de datos en tanto que las teorías, en principio, predicen innumerables resultados empíricos, aun aquellos que en un momento determinado sólo pudieran ser considerados como posibles.

No resulta difícil entender que la tesis de la subdeterminación es una versión moderna de escepticismo radical: No es posible formular un conjunto infinito de afirmaciones a partir de la observación de sólo unos cuantos datos ni tampoco explicar los hechos de una forma única.

En primer lugar, la generalización de esta tesis a cualquier tipo de conocimiento, no necesariamente científico, nos conduce a la aceptación de explicaciones ad hoc de los hechos; la verdad o la falsedad de un enunciado sólo exige la invención de una "historia", no necesariamente basada en la lógica racional.

Hechos como la circulación de la sangre en los animales y la estructura atómica de la materia pueden explicarse desde el punto de vista de teorías científicas con tal evidencia que no es posible explicarlos racionalmente de manera diferente. Del mismo modo, ante 
una explicación única, si las evidencias de la misma no son suficientes, consideramos que no es científicamente válida y procedemos a rechazarla, pues sería posible que una teoría aún desconocida aportara los elementos suficientes para ser aceptada como válida.

En relación con la tesis de kuhn, puede anotarse que, por una parte, los métodos experimentales del siglo XIX no se caracterizaron siempre por la precisión en las medidas de modo que la influencia de la teoría atómica pudo precipitar su aceptación aún antes de su completa verificación. Sin embargo, desde comienzos del siglo XX se han acumulado tantas evidencias, tanto teóricas como experimentales, que, más allá de la duda metódica, no es posible la duda razonable.

El filósofo de la ciencia Tim Maudlin (Sokal y Bricmont, 1999) ha analizado la propuesta kuhniana en dos niveles: En el primero se aducen razones a favor de una tendencia correcta de los debates científicos del siglo XIX y en contra de las comprobaciones que condujeron a su aceptación por su origen no necesariamente científico, en algunos casos, o por ser simplemente insuficientes en otros. En el segundo nivel Kuhn sostiene la tesis del origen no empírico de los cambios de paradigma, que inicialmente condiciona la concepción de las cosas al interior de un nuevo esquema, a la espera de su posterior confirmación a partir del experimento.

El primer nivel de la propuesta de Kuhn se refiere a un hecho histórico cuya discusión no pertenece a las ciencias naturales. En cuanto a la afirmación de kuhn según la cual la percepción de los resultados experimentales está determinada por la teoría, podemos afirmar que es tan desacertada como afirmar, desde una posición diametralmente opuesta, que las experiencias científicas conllevan una interpretación que les es propia.

Más aun, la diversidad y la fuerza de las razones en que se basa una teoría científica determinan el grado de certeza de la misma. En este sentido, los argumentos que sustentan la teoría de la gravitación universal de Newton tienen mayor solidez que los argumentos históricos que establecen las causas del descubrimiento de América y el grado de aceptación de la teoría atómica de la materia, por parte de científicos y no científicos, es mayor que el de la teoría cuántica de la gravedad.

Como escribe el mismo Feyerabend:

"No basta con socavar la autoridad de las ciencias mediante argumentos históricos: ¿porqué motivo sería mayor la autoridad de la historia que la de, pongamos por caso, la física?" (Feyerabend, 1993).

\section{EL RELATIVISMO DE FEYERABEND}

La esencia del pensamiento de este filósofo, desde el punto de vista del relativismo del conocimiento, parece hallar-se en la siguiente afirmación: "Todas las metodologías tienen sus límites y la única regla que sigue siendo válida es: Todo vale” (Feyerabend, 1975).

No es fácil entender a Feyerabend, sobretodo si se tienen en cuenta sus últimos escritos en los que se hallan afirmaciones que parecen contradecir su filosofía del "todo vale", como las que se citan a continuación:

Un anarquista ingenuo dice que: a) tanto las reglas absolutas como las reglas que dependen del contexto tienen sus límites, de lo que infiere que b) todas las reglas y 
todos los criterios carecen de valor y hay que abandonarlos. La mayoría de los críticos me toman por un anarquista ingenuo en el sentido que acabo de mencionar (...) Pero aunque estoy de acuerdo con a) no estoy de acuerdo con b). Lo que pretendo decir es que todas las reglas tienen sus propios límites y que la "racionalidad" global no existe. Nada más lejos de la verdad que querer avanzar sin reglas ni criterios (Feyerabend, 1993).

El hecho concreto, sin embargo, es que dentro de esta ambigüedad, sus frecuentes diatribas contra la ciencia contemporánea y los debates anticientíficos de algunos de sus seguidores, no dejan lugar a duda sobre el carácter relativista de su pensamiento filosófico. Así se muestra en sus reflexiones acerca de Estado y ciencia, en las que se niega a aceptar, para las ciencias naturales, una epistemología realista que él mismo utiliza, por lo menos en forma implícita, en las ciencias humanas:

Es absolutamente necesario aprender física, astronomía, historia. Nadie puede substituirlas por la magia, la astrología o el estudio de las leyendas. Tampoco nos contentamos con una presentación meramente histórica de los hechos y de los principios físicos (astronómicos, históricos, etc.). No se dice que algunas personas creen que la tierra gira alrededor del sol, mientras que otras consideran la tierra como una esfera hueca que contiene al sol, los planetas y las estrellas fijas. Se dice que la tierra gira alrededor del sol; todo lo demás es pura idiotez (Feyerabend, 1975).

En algunos casos, una distinción entre el contexto del descubrimiento y el contexto de la justificación llevarían a entender la postura crítica de Feyerabend:

Nuestro primer paso en la crítica de los conceptos y reacciones habituales consiste en salir del círculo y, o bien idear un nuevo sistema conceptual (...) o bien importar dicho sistema desde el exterior de la ciencia, de la religión, de la mitología, de las ideas de la gente incompetente o de las divagaciones de los locos (Feyerabend, 1993).

Ante todo, la justificación de una teoría no puede ser el resultado de cualquier método: deductivo, inductivo, de analogía, de intuición ni mucho menos de alucinación. Pero el descubrimiento de la misma es un resultado pragmático que justifica el recurso de todos los métodos de investigación posibles.

Aceptada la distinción entre uno y otro contexto, el método crítico propuesto por Feyerabend puede aceptarse si se refiere al contexto de descubrimiento de las teorías. Sin embargo la negación rotunda de tal distinción por parte del filósofo austriaco implica que, independientemente del contexto, no existen códigos de verificación de una teoría; pero el contexto de verificación y el contexto de descubrimiento, aunque en la práctica pueden darse simultáneamente, son distinguibles, por lo menos, en abstracto.

Sokal ilustra, con un ejemplo, cómo 'los procedimientos de justificación de teorías no estarían nunca sometidos a restricciones de orden racional" si esta distinción no fuera posible:

"Pensemos... en las investigaciones policiales: se puede descubrir al culpable como consecuencia de todo tipo de acontecimientos fortuitos, pero los argumentos propuestos para demostrar su culpabilidad no gozan de esa libertad, aun cuando los criterios en cuanto a las pruebas evolucionen históricamente" (Sokai y Bricmont, 1999). 


\section{CONCLUSIÓN}

Las tendencias epistemológicas de Kuhn y Feyerabend apuntan a desconocer la validez universal de las teorías científicas y a considerar el conocimiento científico en un plano de igualdad con el mito. Ambas tendencias comparten un rasgo característico: el relativismo del conocimiento, entendido como una forma de pensamiento filosófico que considera los predicados de verdad y falsedad de una proposición, principio o ley, como dependientes de un individuo o de un grupo humano.

Es evidente que la controversia suscitada alrededor de la subjetividad u objetividad del conocimiento desde la época de los filósofos griegos hasta nuestros días, es el referente común de las corrientes filosóficas que, desde uno u otro lado, tratan los problemas de la ciencia contemporánea. En la práctica, sin embargo, la problemática se traduce en una permanente referencia crítica al empirismo como propuesta epistemológica y a una parodia de ciencia, generalmente basada en los contenidos de textos escolares, no siempre de los mejores, apodada ciencia empírica.

De esta manera se intenta justificar el apriorismo en la enseñanza y en la investigación en el campo de las ciencias naturales y la ausencia de procesos sistemáticos de observación y de experimentación como medios de confrontación de cualquier teoría. Una teoría científica debe apoyarse en pruebas empíricas, pues no basta la coherencia interna ni el uso del lenguaje o del formalismo teórico para ser considerada como discurso científico.

Solamente sobre la base de la distinción entre hechos y creencias consensuales puede desarrollarse el espíritu crítico de los estudiantes, quienes, frente a sus aciertos o desaciertos pueden evaluarse por comparación con los hechos que tienen existencia independiente de sus juicios. Del mismo modo la pedagogía debe llevar al estudiante de la reconocer que, si hechos y descripciones no corresponden a categorías distintas, no sería posible distinguir el error de la verdad en el campo de la ciencia, porque son los hechos los que determinan, en última instancia, el grado de validez y de certeza del conocimiento científico acerca del mundo exterior.

\section{BIBLIOGRAFÍA}

Bertalanffy, L. Von, 1987. Historia y situación de la teoría general de sistemas, en Tendencias en la teoría general de sistemas, Madrid: Alianza Universidad.

Carnap, R., 1986, Fundamentación lógica dela física. Barcelona: Orbis.

Deleuze, G., 1996, Empirismo y subjetividad Barcelona: Gedisa.

Feyerabend, P.,1975, Against Method. Londres: New Left Books.

Feyerabend, P.,1993, Against Method. Londres: Verso, 2ª. Ed.

Hessen, J., 1997. Teoría del conocimiento. México, D. F.: Ed. Porrúa, 10ª Edición.

Kuhn, T., 1990, La estructura de las revoluciones científicas. Madrid: FCE. 
LeShan, L. y Margenau H., 1996, El espacio de Einstein y el cielo de Van Gogh. Barcelona: Gedisa.

Russel, B., 1976, Análisis de la materia. Madrid: Taurus, 2ª . Edición.

Sokal, A. y Bricmont, J., 1999, Imposturas intelectuales. Barcelona: Paidós. 